\title{
GLAD!
}

Revue sur le langage, le genre, les sexualités

03 | 2017

Hétérosexualités

\section{Hétérosexualités en action}

La production des masculinités prédatrices et complices

Heterosexualities in Action. The Construction of Predatory and Complicit

Masculinities

\section{Vulca Fidolini}

\section{OpenEdition}

Journals

Édition électronique

URL : http://journals.openedition.org/glad/737

DOI : $10.4000 /$ glad.737

ISSN : 2551-0819

Éditeur

Association GSL

\section{Référence électronique}

Vulca Fidolini, « Hétérosexualités en action », GLAD! [En ligne], 03 | 2017, mis en ligne le 10 décembre 2017, consulté le 20 janvier 2021. URL : http://journals.openedition.org/glad/737 ; DOI : https:// doi.org/10.4000/glad.737

Ce document a été généré automatiquement le 20 janvier 2021.

\section{(c) (i) (9)}

La revue GLAD! est mise à disposition selon les termes de la Licence Creative Commons Attribution -

Pas d'Utilisation Commerciale - Pas de Modification 4.0 International. 


\section{Hétérosexualités en action}

La production des masculinités prédatrices et complices

Heterosexualities in Action. The Construction of Predatory and Complicit

Masculinities

Vulca Fidolini

\section{Introduction}

1 La recherche sur laquelle porte cet article a été conduite entre 2011 et 2015. Elle visait à étudier l'hétéronormativité à travers l'analyse des constructions de la masculinité et des vécus sexuels d'un groupe de jeunes marocains (Fidolini 2015). Les résultats principaux ont montré que, contrairement à ce que l'on pourrait penser, le pouvoir de l'hétéronormativité et la complémentarité asymétrique qu'elle construit entre masculinité et féminité ne révèlent pas leur force hiérarchisante à travers la seule opposition entre hommes et femmes ou entre hétérosexualité et homosexualité. Ce pouvoir se construit aussi à travers le rapport entre hétérosexualités et entre récits hétérosexuels (Coates 2003), dans les espaces homosociaux où différentes hétérosexualités se confrontent, se défient, sont jugées réciproquement, et sont mises à l'épreuve de la hiérarchie du genre (Flood 2008). Cet article cherchera à analyser ce rapport entre récits hétérosexuels afin de montrer comment s'expriment les formes plurielles du pouvoir hétéronormatif.

2 Les résultats présentés dans ce texte sont issus d'une recherche sociologique qualitative auprès de 23 jeunes hommes marocains, immigrés en France (et plus précisément en Alsace), âgés de 20 à 30 ans, musulmans, arrivés en Europe alors qu'ils avaient entre 13 et 22 ans. Les jeunes interrogés sont issus de milieux sociaux diversifiés, ils proviennent surtout des centres urbains du Maroc et sont, dans la plupart des cas, étudiants au moment des entretiens réalisés.

3 Cette recherche visait également à comparer leurs vécus avec ceux d'une autre population de jeunes marocains rencontrés en Italie. Néanmoins, dans ce texte je présenterai certains résultats du seul terrain d'étude français. L'influence de certaines 
représentations contemporaines, essentialistes, de la masculinité patriarcale et arabomusulmane semble ici beaucoup plus pertinente à analyser en se référant au passé colonial français et à son histoire d'immigration depuis des pays du sud de la Méditerranée.

Les travaux de Lahoucine Ouzgane (2003) ont mis en évidence que, pendant longtemps, les recherches en sciences sociales ayant interrogé les masculinités dans les sociétés islamiques, et notamment dans les pays du sud de la Méditerranée, n'ont fait que reproduire une lecture partielle des dynamiques de l'identification de genre chez les hommes. Les masculinités ont été interprétées à partir de l'étude de la relation asymétrique entre les sexes, qui demande aux hommes d'assurer d'abord la perpétuation de la lignée agnatique en raison de leur pouvoir reproductif hétérosexuel. En ce qui concerne le cas du Maroc et des hommes marocains, le thème des masculinités a fait l'objet d'études récentes ayant déconstruit cette approche essentialiste. Après les travaux d'Abdessamad Dialmy (2009) - qui ont cherché à saisir le « degré de changement des critères définitionnels ordinaires de la masculinité [...] et leur caractère socialement construit " (ibidem, 1-2) dans le Maroc contemporain -, l'attention s'est concentrée surtout sur les masculinités homosexuelles. Les recherches de Gianfranco Rebucini (2009) et celles de Marien Gouyon (2010) sur l'homosexualité au Maroc et chez les Marocains en milieu migrant ont montré que les constructions des masculinités dialoguent de manière complexe avec la définition des identités hétérosexuelles et homosexuelles. Ces recherches ont affirmé l'intérêt d'une démarche d'enquête visant à articuler les questions du genre et de la sexualité afin de décrire les mailles du pouvoir hégémonique de l'hétérosexualité. Néanmoins, elles se sont focalisées surtout sur l'emprise de ce pouvoir sur la construction d'identités nonhétérosexuelles. Contrairement aux études déjà réalisées, l'un des objectifs de ma recherche a été d'interroger directement le pouvoir de l'ordre binaire des sexes et l'injonction à l'hétérosexualité chez des hommes marocains se déclarant hétérosexuels (Fidolini 2017).

\section{Les enjeux méthodologiques de l'étude}

5 La démarche méthodologique adoptée a été caractérisée par la conjugaison de deux techniques d'enquête : les entretiens semi-dirigés et l'observation ethnographique. Il a été fait le choix de ne pas se limiter à l'enregistrement de récits d'entretiens. Les interviews ont été accompagnées d'un long travail d'observation pendant lequel j'ai pu partager avec mes interlocuteurs différents moments de leur vie quotidienne, dans les lieux de la sociabilité ordinaire et festive: soirées en boîte de nuit ou dans les résidences universitaires, repas en famille ou au restaurant, etc. L'intention était de construire un rapport de confiance avec les interviewés - ou, du moins, avec une bonne partie des interlocuteurs - afin de pouvoir traiter le thème de la sexualité plus aisément, au sein d'un entretien enregistré.

6 Cette immersion ethnographique caractérise mon approche de recherche et d'analyse. C'est aussi la raison pour laquelle dans ce texte j'utilise souvent des termes comme copines ou filles, qui reprennent directement les expressions utilisées par les jeunes hommes rencontrés. L'analyse du langage corporel occupe aussi une place centrale dans ma démarche. L'observation ethnographique a suivi l'expression changeante des corps masculins en permettant d'en sonder les manifestations plurielles dans différents 
contextes d'interaction. Un « journal de bord » a été tenu. Je ne l'utilisais pas pendant les observations, craignant de perturber la (prétendue) naturalité des événements. Au contraire, je me suis servi à plusieurs reprises de mon téléphone mobile pour noter in situ ce que j'observais et revenir en détail sur les différents points retenus lors de la transcription.

7 Favorisé aussi par le fait de mener un terrain avec des pairs, des jeunes hommes ayant plus ou moins mon âge, je n'ai pas rencontré de difficulté particulière pour traiter la question de la sexualité en entretien. Néanmoins, comme j'ai pu l'expliquer ailleurs (Fidolini 2017), cela ne m'empêche pas de reconnaître qu'une telle facilité a aussi été porteuse d'obstacles interprétatifs (Bozon 1995). Ces obstacles ont notamment concerné l'usage que les interviewés font de la religion, et notamment de la référence à l'islam. Par exemple, lorsque je posais des questions au sujet des pratiques de sexe anal ou oral adoptées par les interviewés, souvent les réponses se cachaient derrière la nette distinction entre une sexualité conjugale licite et sacrée, où le sexe oral et le sexe anal étaient considérés « inadmissibles » dans le respect des préceptes islamiques (Bouhdiba 1975), et des relations non conjugales illicites, où la sexualité (qui serait interdite) semblait pouvoir permettre un répertoire de pratiques sexuelles paradoxalement plus large. Ce voile normatif qui inspirait la rhétorique de mes interlocuteurs a parfois caché les "vrais» vécus des interviewés. Cela m’a paru particulièrement évident lorsque l'un des jeunes hommes interviewés a révélé son homosexualité seulement au cours d'un deuxième entretien, qui a eu lieu presque deux ans après une première conversation enregistrée. Au cours de cette première interview, le jeune homme en question s'était dit hétérosexuel (Fidolini 2017). Comme il put me l'expliquer ensuite, le fait d'avoir utilisé pendant longtemps la référence à l'islam pour raconter sa sexualité lui avait permis de ne pas devoir justifier sa conduite ni voir son orientation sexuelle questionnée, notamment face à ses parents et à ses amis. En se référant à l'islam, et en affirmant avec conviction être un "musulman pratiquant ", la seule sexualité possible ne pouvait être qu'hétéronormative et conjugale.

De plus, cette même référence aux préceptes de l'islam était aussi utilisée par mes interlocuteurs en tant qu'appui rhétorique pour négocier une sorte d'authenticité culturelle en milieu migrant, à l'étranger, et cela notamment vis-à-vis de la population majoritaire, voire du chercheur lui-même - jeune homme, européen, non marocain et non musulman. Comme le dit Safiq (24 ans, étudiant universitaire résidant à Strasbourg, originaire d'une famille aisée de Casablanca) :

9 Avec une Arabe [par rapport à une relation avec une jeune femme française] c'est quand même un niveau différent. Ne prends pas ça comme une discrimination hein [il s'adresse à moi], c'est culturel, pour moi il y a beaucoup plus de règles religieuses dans les pays arabes qu'en Europe. C'est un truc culturel c'est pas que la fille, il y a autre chose, il y a une famille derrière. Et moi je me projette ça, voilà, c'est ma famille, c'est ma cousine, c'est machin, et même ses parents à elle. Les parents, il y a rien de plus triste pour eux que de voir leur fille bafouée.

La référence à une prétendue " culture arabe », « marocaine », « musulmane » est alors mise en scène et contribue en quelque sorte à essentialiser le profil de ces jeunes hommes qui cherchent à se différencier des " autres ", à savoir « les Européens ", "les Français » (Fidolini 2014). 


\section{Une analyse des formes d'expression de l'hétéronormativité}

11 Dans cette recherche, en suivant la réflexion de Judith Butler, l'hétéronormativité a été interprétée comme le modèle hégémonique des rapports de genre qui postule la complémentarité asymétrique des sexes et la primauté de l'hétérosexualité. J'entends l'« hégémonie » au sens gramscien, et donc comme une forme de domination fondée sur « la combinaison de la force et du consentement qui s'équilibrent de façon variable, sans que la force l'emporte par trop sur le consentement, voire en cherchant à obtenir que la force apparaisse appuyée sur le consentement de la majorité » (Gramsci 2012 : 234). Cette domination ne s'exprime pas nécessairement à travers une forme de pouvoir violent et visible, mais plutôt par l'expression d'un contrôle constant et attractif.

12 Comme l'affirme Butler, le pouvoir de l'hétérosexualité se nourrit des tentatives subjectives de confirmer ce contrôle attractif: "L'imitation est au cœur du projet hétérosexuel et de ses binarismes de genre [...], l'hétérosexualité hégémonique est ellemême un effort constant et répété d'imitation de ses propres idéalisations » (Butler 2009: 133). Ainsi, son pouvoir produit les actions subjectives présupposant (et performant) le rapport causal entre le sexe, le genre et le désir. Or, si Butler nous a notamment montré comment ce rapport causal peut être subverti, ma démarche d'étude a été caractérisée - au contraire - par la tentative d'observer empiriquement comment les hommes cherchent à construire et à imiter ce rapport causal. L'objectif est donc de ne pas focaliser l'analyse de l'hétérosexualité sur son caractère de norme oppressante " pour la majorité, certes, mais principalement pour ceux et celles qui ne s'y conforment pas » (LeBrun $2003: 13$ ), mais d'en questionner plutôt l'impact normatif sur ceux et celles qui cherchent à s'y conformer.

Ainsi, en accordant une place centrale à l'analyse des récits et des mises en scène des corps masculins dans différents contextes homosociaux, entre hommes, cet article essaie de décrire comment la construction de la masculinité hétérosexuelle - hégémonique (Connell 2005) - coïncide avec la définition d'autres profils masculins «complémentaires» qui participent à reproduire le pouvoir de l'hétéronormativité. Plus précisément, je m'attacherai à analyser comment ce que je nomme la "masculinité prédatrice »-caractérisée par une hétérosexualité active et débordante - s'exprime par la production consubstantielle d'autres masculinités « complices» (Connell 2005 : 79). Comment la masculinité hétéronormative se configure-elle alors en tant que représentation hégémonique à confirmer et à imiter chez les jeunes rencontrés ? Comment, au sein de différents cadres d'interaction (homosociaux et non homosociaux), la production de cette masculinité hétéronormative construit-elle les conduites d'acteurs " complémentaires » qui en soutiennent le rôle dominant, et qui deviennent complices d'un modèle hégémonique de référence?

\section{La masculinité prédatrice. Une question de regards}

14 Quand je parle de masculinité prédatrice, je fais notamment référence au processus de biologisation de la masculinité par lequel le rôle des hommes est réduit à l'expression de leurs instincts et de leurs désirs (hétéro)sexuels envers les femmes. Ces instincts finissent par être considérés comme un trait essentiel de la masculinité, une caractéristique inaliénable de sa « nature » (Ferrero Camoletto \& Bertone 2009). 
Comme le souligne Franco La Cecla (2002), ce processus de biologisation est l'effet d'une compétition entre hommes et a pour but le contrôle de la sexualité des femmes. Cette représentation (essentialiste) est souvent utilisée pour désigner les masculinités des pays de la rive sud de la Méditerranée, tout comme celles de certains pays de la rive nord, comme l'Italie, l'Espagne, la Grèce (idem). La masculinité prédatrice participe à définir le rôle de l'homme au sein du système patriarcal. Dans ce cadre, la femme est érigée en symbole de la pudeur et de la pureté - avant tout sexuelle - qui fait de la figure féminine la vraie dépositaire de la respectabilité masculine, et notamment de l'honorabilité des hommes vis-à-vis des autres hommes. Le modèle de la masculinité qui est véhiculé exalte la virilité, l'orientation hétérosexuelle de l'homme, sa capacité reproductive, mais aussi ses capacités à contrôler la sexualité des femmes, mettant en scène tout un ensemble de qualités dont le «vrai » homme ne peut pas être dépourvu, notamment aux yeux des pairs.

Pendant les différentes phases de ma recherche de terrain - qui, il est important de le préciser, m'a permis d'observer ces jeunes hommes dans leurs relations avec d'autres immigrés, avec des Marocains et Marocaines descendant-e-s d'immigrés, voire avec des personnes faisant partie de la population majoritaire - j'ai souvent eu l'impression que mes interlocuteurs mettaient en scène cette hétérosexualité, débordante et indispensable, et cette attitude prédatrice, de manière stratégique. Cette mise en scène leur permettait de répondre aux prétendues attentes des pairs, moi y compris, vis-à-vis de leur conduite sexuelle. Les regards d'autres hommes, dans l'espace homosocial, construisaient donc la scène privilégiée pour la reproduction de cette biologisation de l'hétérosexualité masculine. Surtout lorsque je rencontrais pour la première (ou les premières) fois ces jeunes hommes, la mise en scène de la masculinité prédatrice se configurait comme l'étape préliminaire utilisée afin d'établir le premier contact (Kiesling 2005). Il s'agissait de construire une relation entre inconnus, trouver des terrains d'entente, de comprendre et d'interpréter l'autre : qui étais-je? Quelle était mon orientation sexuelle à leurs yeux et, inversement, la leur aux miens? Par exemple, lorsque, avec Fouad (21 ans), j'ai rencontré pour la première fois son groupe d'amis marocains de Mulhouse - tous étudiants à l'université comme lui -, après avoir raconté - de manière très générale - que je travaillais à une thèse sur les masculinités des jeunes Marocains, la réaction fut :

Ah tu vas t'amuser! [...] Tu verras des Marocains qui boivent et des Marocains qui ne boivent pas, qui fument et qui ne fument pas, des musulmans qui prient et qui ne prient pas. Mais quelque chose tu la trouveras partout: les Marocains, tous les Marocains, aiment baiser les filles !

Cette naturalisation du désir sexuel était utilisée de manière différente en fonction des situations. Si dans le cas des amis de Fouad il s'agissait d'une phrase lancée pour rechercher aussi l'approbation des autres jeunes hommes (trois, au total) présents dans la chambre de la résidence universitaire où j'étais, dans d'autres cas la mise en scène de ce récit masculin prédateur était utilisée pour marquer, dès le début, une hiérarchie des masculinités entre l'interviewé et moi-même. Cela, par exemple, m'est arrivé avec Moussa (21 ans, Strasbourg, jeune homme au chômage au moment de l'entretien, issu d'une famille d'origine modeste) qui, avant de commencer l'entretien dans un café du centre-ville de Strasbourg, me demanda si l'on pouvait faire l'interview assis à la 
terrasse du café alors que j'avais proposé d'entrer pour mieux suivre son récit loin du bruit :

Question : Si tu veux on peut s'asseoir derrière, j'ai vu il y a de la place...

Moussa : Derrière ?! ? Derrière on va pas regarder les filles ! [...] ça ne me dérange pas si tu veux on se pose là-bas, moi ça me dérange pas, tu me poses n'importe quelle question je te réponds, je sais que c'est important pour toi ton truc [l'entretien]. Mais moi je suis là [en indiquant l'intérieur du café, le lieu de l'entretien] et mes yeux sont là-bas [en indiquant la place en face du café]!

Selon un lieu commun très répandu, la masculinité prédatrice s'exprimerait notamment dans les espaces publics : c'est là que l'acteur masculin pourrait exhiber sa puissance virile hétérosexuelle et affirmer son rôle vis-à-vis d'autres masculinités concurrentes (Whitehead 2002: 116; Saadi-Mokrane 2004). Il ne s'agit pas vraiment de pratiquer l'hétérosexualité mais surtout de l'afficher en tant que modèle de référence, à mettre en scène de façon permanente, régulière et visible afin de confirmer aux yeux des autres que l'on est bien des hommes, des « vrais » (Flood 2008 ; Gourarier 2017). En ce sens, le profil masculin prédateur devient l'un des rôles à jouer pour confirmer l'ordre du genre hétéronormatif et sa hiérarchisation des formes d'expression du masculin et du féminin (Clair 2008).

Or, cette construction de la masculinité chez les populations immigrées ou descendantes d'immigrés du Maghreb a souvent été abordée, en France, en analysant la logique de l'hypervirilisation $\mathrm{du}$ corps masculin. La critique $\mathrm{du}$ concept d'hypervirilisation a montré comment l'(hétéro)sexualité, envisagée comme incontrôlable, finirait par se traduire dans le regard majoritaire - et selon une lecture culturalisante - en tant que seule réponse possible de la part de ces populations à leur condition minoritaire, subalterne et marginalisée (Guénif-Souilamas 2004). Comme le souligne Nacira Guénif-Souilamas, en France, les représentations du "garçon arabe " sexuellement dangereux pour les filles se sont cristallisées dans l'imaginaire collectif, en durcissant l'opposition grossière entre les prétendus « archaïsmes » des populations originaires des ex-colonies d'une part et la prétendue «modernité » républicaine de l'autre (idem).

Dans ce texte, je souhaiterais m'éloigner de ces représentations de l'homme immigré hypervirilisé et racisé, ainsi que des assignations majoritaires et culturalistes dont il fait l'objet, pour interroger plutôt les modalités de négociation de ces mêmes représentations de la part des interviewés. Les images de la masculinité hétérosexuelle débordante, souvent essentialisée par la référence au profil des hommes immigrés de la rive sud de la Méditerranée (La Cecla 2002), peuvent être renégociées et agencées différemment en fonction des situations et des interlocuteurs, se révélant plus ou moins influentes et contraignantes sur les formes de mise en scène de la masculinité des membres des populations minoritaires. De surcroît, les interviewés ont montré avoir conscience des attentes qui existent autour des formes d'expression de leur masculinité : ils participent tantôt à renforcer ces attentes, tantôt à les délégitimer, tout en produisant une hiérarchie des masculinités interne au groupe minoritaire, qui ne concerne donc pas seulement le rapport entre populations majoritaire et immigrée. Ainsi, par exemple, la référence implicite ou explicite à l'islam en tant qu'expression de la foi de l'interviewé, à un modèle patriarcal de la masculinité, ou à la sexualité des femmes entendue comme dépositaire de la réputation masculine, peuvent devenir des 
outils pour construire différentes masculinités. Cela permet aux interviewés de négocier leur place vis-à-vis du regard des autres, en fonction des cadres d'interaction et des sujets qui y sont impliqués.

\section{Le corps : objet et agent de la masculinité sous le regard d'autrui}

21 Houcine (26 ans), Mehdi ( 26 ans) et Soufiane (26 ans) sont tous trois originaires de la même ville du Maroc, Biougra. Ils se sont retrouvés, au même âge, à Strasbourg, chacun suivant un parcours biographique différent. Houcine, jeune homme issu d'une famille aisée, est arrivé seul en Alsace à l'âge de 17 ans pour ses études universitaires en économie. Mehdi, étudiant universitaire en économie à Agadir, issu d'une famille de la classe moyenne, l'a rejoint quelques années après, lorsqu'il avait 22 ans, pour terminer sa formation en France. Soufiane est arrivé un an plus tard - à 23 ans -, pour chercher du travail, venant d'Italie où il avait émigré avec ses parents à l'âge de 17 ans. Soufiane, issu d'une famille d'ouvriers, avait obtenu un diplôme professionnel en Italie (l'équivalent d'un brevet d'études professionnelles des métiers de la restauration et de l'hôtellerie en France) et était arrivé seul à Strasbourg.

Les trois jeunes hommes - avec qui j'avais déjà pu passer plusieurs soirées m'attendaient chez Houcine. C'était un vendredi soir. Soufiane m'avait proposé d'aller manger ensemble dans un restaurant marocain qui était une sorte de self-service géré par deux femmes marocaines que Soufiane connaissait. Il s'agissait d'une mère, la cinquantaine, et de sa fille, âgée de 24 ans. La mère, qui gérait le local, avait suivi en France son mari émigré, et sa fille était née à Strasbourg. Nous entrâmes dans le restaurant vers 21 heures alors qu'il n'y avait personne. Le comptoir du self-service coupait la salle en deux : d'un côté les clients, de l'autre les deux femmes qui géraient le restaurant. Pendant que nous discutions sur le choix des plats à commander, je me rendis compte que les deux espaces du restaurant établissaient la configuration de l'interaction. La nette séparation entre clients et gérantes se révélait fonctionnelle à la construction de deux sphères homosociales distinctes: entre hommes d'un côté et entre femmes de l'autre. Soufiane semblait particulièrement à l'aise dans cette situation et n'hésita pas à occuper le devant de la scène. Ainsi je décidai de m'éloigner un peu avec Mehdi - le plus timide du groupe - pour observer les actions de Soufiane, et d'Houcine également, qui le suivait de près. La présence de Mehdi fut fondamentale car, en l'ayant à mes côtés, je pouvais lui demander de me traduire certains mots arabes que Soufiane et Houcine utilisaient pour parler avec la mère de la fille.

Soufiane commença à raconter à la mère marocaine qu'il était en train de suivre une formation professionnelle et qu'il aurait aimé, lui aussi, travailler dans la restauration. Il semblait vouloir mettre en valeur le fait qu'il était un garçon sérieux. Soufiane répétait souvent qu'il n'avait pas de copine et qu'il attendait de rencontrer la « femme de sa vie » pour se marier. Il expliquait ne pas aimer les boîtes de nuit et ne pas vouloir perdre son temps dans des "vices" comme l'alcool, les cigarettes, les filles. Il promouvait sa masculinité en la différenciant d'autres profils subalternes du jeune immigré en situation précaire, aux mauvaises fréquentations et à la mauvaise réputation. Il s'adressait toujours à la mère - qui l'écoutait un peu amusée - alors qu'Houcine confirmait au fur et à mesure ce que Soufiane venait de dire pour soutenir le récit de son ami. 
Après cette première phase de la conversation, ce fut la mère qui décida de relancer la discussion avec Soufiane à travers des provocations plus ou moins directes. Elle soulignait que sa fille était potentiellement une "bonne épouse » en raison du fait qu'elle tenait beaucoup à sa famille, à son père et à ses frères, qu'elle avait une prédisposition à s'occuper de la maison car, tout en continuant les études, elle l'aidait pour faire le ménage. Finalement, la mère ajouta que sa fille n'avait pas encore de copain. Néanmoins, son discours n'était pas direct puisque, dans son récit, elle s'appuyait toujours sur la référence aux habitudes des femmes dites "européennes", en y opposant le comportement différent de sa fille. La mère soulignait un prétendu désengagement des femmes européennes des tâches domestiques et de la religion pour faire entendre, à l'inverse, que sa fille n'était pas du tout comme cela, et qu'elle tenait beaucoup à "la valeur de la famille» comme toute "bonne musulmane». La figure de la femme était associée à l'accomplissement d'un "rôle domestique ", essentialisé, interne à la famille, faisant référence à la dimension du « dedans » (Bourdieu 2000) qui soutenait une configuration familiale patriarcale et figée. Aussi bien la mère marocaine que Soufiane et Houcine reproduisaient la rhétorique de l'opposition entre leurs origines et les habitudes de la société d'accueil et de la population majoritaire afin de négocier autrement leur condition de "minoritaires». Houcine me confirma ensuite que cette mère était clairement en train de "faire de la publicité » pour sa fille car, à son avis, le fait que la jeune femme était encore à la maison à l'âge de 24 ans pouvait être ressenti comme une honte de la part de cette mère, notamment vis-à-vis d'autres jeunes hommes marocains. Autrement dit, selon Houcine, la mère voulait justifier le fait que sa fille n'avait pas encore trouvé un mari.

Nous avions commencé à manger et la mère, d'un ton à la fois sérieux et amusé, poussa encore plus loin la conversation en faisant entendre à Houcine qu'un mariage avec sa fille lui aurait permis d'avoir une très belle fête avec "beaucoup de nourriture fait maison " puisqu'elle-même aurait pris en charge l'organisation de l'événement. La mère de la jeune femme, en plaisantant, répétait, en référence à l'organisation du mariage et au prix de la nourriture :

Mon pauvre! T'es venu ici pour manger à 5 euros, tu t'en vas avec une addition de 2000 euros!

C'était justement la relation entre propriétaire et client qui permettait ces allusions, car l'interaction restait liée à son cadre formel, aux rôles des acteurs et aux espaces séparés où se déroulait l'interaction. L'arrivée d'autres personnes dans le restaurant interrompit la conversation, obligeant Soufiane et Houcine à s'éloigner du comptoir pour permettre aux deux femmes de continuer leur travail.

En sortant du restaurant, le scénario changea rapidement. Nous étions désormais dans un espace homosocial exclusivement masculin, et le profil prédateur de la masculinité avait pris le devant de la scène. Houcine affirmait ainsi :

T'as vu la mère comme elle me regardait, t'as vu ? Ben Soufiane, toi tu prends la fille, moi je prends sa mère!

L'attitude respectueuse montrée par Soufiane, et le fait d'être tombé dans le piège des blagues de la mère marocaine, coûtaient à ce jeune homme les railleries de son ami Houcine. Nous étions en chemin vers le centre-ville, qui était juste à quinze minutes de marche, et la rue se transformait en terrain privilégié pour la "vengeance» de 
Soufiane. En évoquant son idole sportive, Cristiano Ronaldo - à qui ses amis disaient qu'il ressemblait en raison de sa coupe de cheveux et de son style de jeu au football -, Soufiane mettait en scène son attitude prédatrice et son rôle de "séducteur de trottoir » (Bologne 2010:268). En arrangeant le col de sa chemise blanche, Soufiane faisait semblant d'ajuster ses cheveux courts et noirs, coiffés avec du gel effet mouillé. Il imitait sa vedette sportive (Duret 1999), se dirigeant vers deux filles qui étaient en train de manger dans un restaurant aux portes du centre-ville. Suivi par Houcine, Soufiane s'arrêta devant la vitrine du restaurant dans lequel dînaient les deux jeunes femmes, très discrètement, dans un coin du local. Houcine et Soufiane se mirent à leur montrer par la vitre leur numéro de portable écrit sur l'écran de leur smartphone, en leur demandant de faire la même chose avec les leurs. Soufiane les invitait à sortir et à continuer la soirée avec nous, pendant que Houcine se rapprochait de la porte principale du restaurant pour essayer d'entrer. Le profil du bon garçon mis en scène face à la mère marocaine et à sa fille avait été complètement remplacé par la mise en scène d'une masculinité prédatrice par laquelle Soufiane créait une syntonie avec son ami Houcine. Notre présence insistante hors du restaurant fatigua bientôt le gérant qui, après quelques minutes, en sortant, nous invita à ne pas déranger ses clients et à ne pas stationner devant la vitrine.

\section{Mises en scène et complicités hétérosexuelles} joués à partir de la mise en scène d'un ensemble de représentations normatives (partagées) de la masculinité par lesquelles chacun s'inscrivait dans l'espace homosocial en étant reconnu par les autres à travers sa conduite. Les relations homosociales étaient donc un lieu d'expression des masculinités, mais aussi de contrôle de ces formes d'expression entre amis (Flood 2008; Anderson McCormack 2014). Ces jeunes hommes, acteurs et spectateurs de leurs performances, se retrouvaient assujettis et dépendants de modèles masculins incorporés. Ces modèles étaient mis en scène pour échapper aux moqueries des autres hommes ou pour gérer la relation avec les femmes, à la fois en se conformant et en négociant les représentations stéréotypées de la masculinité dragueuse et prédatrice.

Cette dialectique de mise en scène de différents modèles et représentations hégémoniques de la masculinité hétéronormative, dans l'espace homosocial et à travers le corps, nous rappelle la notion de "pratique corporelle réflexive" (body-reflexive practice) élaborée par Raewyn Connell (2005: 65). Les stratégies et les techniques du corps par lesquelles les hommes performent les modèles de la masculinité ne dépendent pas exclusivement de la volonté du sujet qui les performe. Selon Connell, elles dépendent également des attentes construites par les autres envers les actions du sujet qui performe un certain modèle masculin. Il peut donc arriver que le sujet qui est l'acteur de la mise en scène de la masculinité devienne dépendant de sa propre performance, parce qu'il connaît les attentes de ses interlocuteurs vis-à-vis de sa mise en scène et qu'il décide, ainsi, de s'y tenir. Chez les jeunes hommes interrogés, la manière d'agencer la mise en scène de la masculinité hétéronormative varie en accord avec les règles de l'espace homosocial ou de la relation avec les femmes ou, encore, des deux scénarios en même temps. Ainsi, les interviewés développent de véritables techniques du corps - «les façons dont les hommes [...] savent se servir de leur corps » (Mauss 1950 : 365) - à travers les différentes formes d'occupation des espaces, à travers 
les techniques de drague, etc., en faisant de leur corps à la fois l'agent de leurs masculinités et l'objet des attentes dont ces mêmes masculinités sont porteuses.

Les mises en scène de la masculinité deviennent un enjeu de hiérarchisation des rapports entre les sujets qui participent aux interactions. Le dialogue entre Soufiane et la mère marocaine dans le restaurant offre un exemple de cette hiérarchisation en termes de rapports extérieurs à l'espace homosocial, dans un contexte de mixité ; les railleries d'Houcine envers Soufiane en offrent une perspective plutôt interne (Demetriou 2015) au groupe homosocial. Dans le restaurant, la mère marocaine jouait un rôle de complice, plus ou moins volontaire, pour la production des masculinités d'Houcine et de Soufiane. Elle provoquait l'expression de la masculinité sérieuse de Soufiane et participait à élaborer aussi le scénario pour la mise en scène de l'attitude railleuse d'Houcine envers son ami Soufiane. Mehdi et moi étions également une partie intégrante de cette dialectique. Les profils de Soufiane et Houcine se configuraient dans le cadre de la soirée décrite en tant que figures hégémoniques en raison du fait que mon rôle d'observateur, ainsi que celui de Mehdi - beaucoup plus timide qu'Houcine et Soufiane, et toujours à mes côtés à l'écart du devant de la scène -, avaient une place complémentaire dans l'action des masculinités protagonistes: nous en étions les spectateurs complices et nous participions à en légitimer la mise en scène homosociale. C'est exactement sur l'importance du rôle complice des pairs dans la production des masculinités prédatrices que je me focaliserai dans la prochaine partie.

\section{Figures complices de la masculinité hégémonique}

Selon la définition qu'en donne Raewyn Connell, les masculinités complices participent à légitimer et à soutenir (par consentement ou à travers son imitation) la masculinité hégémonique (Connell 2005 : 79). Dans le cas d'étude qui sera présenté dans cette partie du texte, l'affiliation marocaine des jeunes impliqués a une place moins importante dans la construction de la masculinité par rapport au cas de Soufiane et de ses amis. Au contraire, l'observation des conduites des jeunes hommes dans les espaces homosociaux révélera des configurations masculines, prédatrices et complices, parfaitement courantes aussi chez d'autres jeunes hommes, de toutes origines, migrants ou pas. Cela permettra de montrer que l'étude des vécus et des récits des jeunes interviewés dans cette recherche n'est qu'un point d'observation, parmi bien d'autres possibles, pour interroger le pouvoir de l'hétéronormativité et ses multiples formes d'expression sur les constructions sociales de la masculinité.

Anouar (25 ans, Illkirch-Graffenstaden), originaire d'Agadir, est issu d'une famille de la classe moyenne. Étudiant universitaire, il est arrivé en France avec ses parents à l'âge de 17 ans. Jeune homme très timide, je l'ai connu au début de mon enquête ethnographique à Strasbourg, en décembre 2011, et j'ai continué à le fréquenter de manière régulière jusqu'en avril 2013.

Avec Anouar j'ai pu construire un rapport de confiance à travers plusieurs étapes. Si, pendant les premiers mois de mon terrain, notamment entre décembre 2011 et février 2012, je l'appelais souvent pour passer des après-midi à la cafétéria de la faculté d'économie avec ses amis - aussi bien français que marocains, descendants et immigrés -, par la suite, Anouar a lui-même commencé à me chercher régulièrement. Un jour, en avril 2012, il avait un rendez-vous avec une jeune femme rencontrée pendant une séance de ses cours de danse latino-américaine. Les deux jeunes s'étaient 
donné rendez-vous pour sortir ensemble, mais la jeune femme n'était pas venue. Déçu, et sachant que je n'habitais pas loin de l'endroit où les deux auraient dû se rencontrer, Anouar m'avait appelé pour me demander si je voulais bien le retrouver " pour parler un peu ». Pendant les deux heures que nous passâmes ensemble, Anouar me raconta librement toutes ses expériences avec les filles, ses difficultés pour construire une relation stable à laquelle il songeait depuis quelque temps, sa passion pour la danse latino-américaine à travers laquelle il avait pu combattre sa timidité et sa peur d'approcher les femmes. Craignant de ne pas être à la hauteur de la situation dans les rencontres avec les jeunes femmes - "je ne sais pas comment il faut faire exactement, je n'arrive pas à en garder une!", me disait-il -, Anouar était surtout très préoccupé du jugement des pairs sur sa conduite. Il semblait toujours chercher l'approbation de ses amis mais, en même temps, ne se reconnaissait pas vraiment dans leurs masculinités à l'attitude prédatrice. Il jouait plutôt le rôle de complice : il s'agissait effectivement du modèle hégémonique qu'il cherchait à reproduire, mais il ne se sentait pas vraiment capable d'en mettre en pratique les traits.

J'avais eu une première démonstration de cette attitude complice d'Anouar lors d'une rencontre entre lui et un autre interviewé, Fouad (21 ans, Mulhouse, jeune étudiant universitaire), un après-midi au centre-ville de Strasbourg. Je me promenais avec Anouar et nous rencontrâmes Fouad, qui était avec des amis marocains. Anouar resta un peu en arrière en observant Fouad parler. Ensuite, une fois repris notre chemin, il me dit :

Tu vois les jeunes comme lui, ça se voit tout de suite qu'il est plein de filles. Il a la tchatche, il sait comment faire avec les filles. J'aimerais bien me débloquer un peu, faire comme lui [...].

La difficulté de ce jeune homme à mettre en pratique une attitude moins timide vis-àvis des femmes avait une place encore plus importante dans les logiques homosociales au sein de son groupe d'amis. Même si, depuis trois ans, Anouar habitait seul dans un studio à Illkirch-Graffenstaden (une commune du sud de Strasbourg), ses amis étaient restés surtout ceux des premières années en France, passées dans le quartier de Cronenbourg, où ce jeune homme avait habité avec ses parents et sa sœur jusqu'à l'âge de 21 ans. Ses copains habituels étaient Mehmet, un jeune d'origine turque arrivé en France pendant l'adolescence, et Fred, un jeune martiniquais.

Comme j'ai pu l'observer au cours de nombreuses occasions, Mehmet (26 ans) avait un rôle dominant dans les dynamiques du groupe. Sa réputation de tombeur de femmes et l'exhibition de sa masculinité prédatrice n'étaient pas seulement affichées par Mehmet - j'avais pu observer qu'il avait l'habitude de toujours sortir entouré de plusieurs filles et fumait souvent ses cigarettes assis sur le capot de sa voiture -, mais sa position hégémonique était aussi légitimée et soutenue par ses amis. Anouar et Fred avaient une sorte de vénération pour lui, pour son style de vie et son attitude avec les femmes, et ils craignaient profondément ses jugements sur les filles auxquelles ils s'intéressaient.

\section{La masculinité prédatrice comme code relationnel dans l'espace homosocial}

Lors d'une soirée organisée par Mehmet dans une boîte de nuit de Kehl ${ }^{1}$, Anouar avait invité une jeune femme d'origine sénégalaise, rencontrée à l'université, et m'avait 
demandé de l'accompagner en voiture pour aller la chercher avant de partir, tous ensemble, vers notre destination. Pendant le trajet, avant de rencontrer son amie, Anouar avait pu me raconter qu'il était très intéressé par cette jeune femme, mais qu'il ne savait pas si elle aurait pu plaire aussi à Mehmet.

C'est une black, tu vas voir, elle est belle [...]. Mehmet n'est jamais content des filles que je trouve, il veut toujours donner son avis, je ne sais pas ce qu'il en pense d'une black. Anouar gérait sa vie, qu'il avait l'impression d'être en train de gaspiller l'argent de ses parents en négligeant les études, et que sa frustration aurait trouvé une solution seulement en construisant un rapport stable avec une jeune femme, «sérieuse ». Mais le jugement de son ami Mehmet reprit bientôt le dessus sur son discours. Anouar ne tarda pas à me raconter que Mehmet lui avait conseillé de ne pas perdre son temps avec la «black» et de continuer à s'amuser pour le reste de la soirée.

était l'importance qu'Anouar attribuait à l'avis de son ami, qui le rendait en même temps dépendant et (co)responsable de la production du modèle hégémonique de la masculinité prédatrice que Mehmet incorporait. Le seul code pour établir une relation avec son copain était celui qui conduisait à imiter - de manière toujours partielle et inaboutie - ce modèle prédateur. Ainsi, le rôle complice et dépendant d'Anouar contribuait à cristalliser la hiérarchie des rapports en jeu, en renforçant l'impact hégémonique de la masculinité de Mehmet. Le script prédateur était en quelque sorte le seul code de la relation homosociale, auquel Anouar semblait totalement subordonné. Refuser ce code, en effet, signifiait abandonner aussi les règles de la relation entre pairs, imposées par Mehmet.

44

Cependant, comme j'ai déjà pu le préciser, Anouar n'était pas le seul à vivre ce rapport de dépendance-complicité vis-à-vis de la masculinité hégémonique de Mehmet. Lors d'une autre soirée passée avec Anouar et Fred ( 24 ans), je pus me rendre compte encore plus clairement de l'emprise du profil hégémonique de Mehmet sur les relations 
homosociales au sein de ce groupe d'amis. J'étais dans la voiture d'Anouar avec Fred, devant l'habitation de ce dernier dans le quartier de Cronenbourg, à Strasbourg, où il habitait avec sa copine. Avant de partir vers le centre-ville, Fred commença à raconter qu'il en avait marre de sa vie de couple et qu'il se sentait emprisonné à la maison avec elle :

Elle m'a soûlé mec, tout le temps elle demande où je vais, avec qui je sors, quand je rentre. Elle ne voulait même pas que je sors ce soir, elle dit qu'elle a peur toute seule à la maison. temporairement renversée. Mais, finalement, le résultat de l'attitude d'Anouar et de Fred était encore une fois voué à reconfirmer le rôle hégémonique de Mehmet et l'importance attribuée à son jugement dans la dynamique homosociale. Les masculinités d'Anouar et de Fred, ainsi que leur mise en scène du modèle prédateur, semblaient nécessiter l'approbation de Mehmet. La légitimation de leur masculinité virile dépendait de l'avis du jeune turc qui, à l'autre bout du fil, complimentait Fred pour sa performance. La reproduction du modèle de la masculinité prédatrice subordonnait la position d'Anouar (et de Fred aussi) vis-à-vis de Mehmet même lorsqu'il ne jouait pas son rôle dominant de manière active et visible. Ce processus faisait d'Anouar une figure complice du rôle hégémonique de son ami Mehmet, tout en obligeant également ce dernier à rester fidèle à son rôle de prédateur, sur lequel il avait construit sa masculinité dans l'espace homosocial.

\section{Observations conclusives}

Dans son anthropologie des masculinités au Maroc, Gianfranco Rebucini (2009) analyse finement la dialectique de double dépendance dans laquelle l'acteur masculin est pris lorsqu'il performe son identification de genre vis-à-vis du regard des autres. Son 
enquête - qui interroge les constructions de la masculinité par l'étude des pratiques homoérotiques et homosexuelles dans la ville de Marrakech - révèle à quel point l'acteur de la masculinité est amené à se confronter, de manière permanente, d'une part aux traits du modèle masculin qu'il souhaite performer et, d'autre part, au regard des spectateurs auxquels sa performance s'adresse. En reprenant la métaphore théâtrale d'Erving Goffman (1987), l'attention de Rebucini est concentrée non seulement sur le rôle du sujet qui performe sa masculinité, mais aussi sur sa capacité à capter le regard des autres pour qu'ils deviennent son public. Il s'agit donc de se focaliser sur l'échange qui se produit entre l'expression de la performance de la part de l'acteur et l'impression de ceux et celles qui assistent à la scène (Rebucini 2009 : 217).

50 La dépendance d'autres acteurs et spectateurs est un repère qui permet au sujet de jouer son rôle en puisant dans un ensemble de représentations communes et d'attentes réciproques. Les cas d'étude analysés affirment le rôle central des processus d'imitation de l'hégémonie de l'hétérosexualité (Butler 2009) au sein des constructions de la masculinité qui reproduisent le modèle prédateur. Ces imitations semblent accompagnées d'un souci constant chez ces jeunes hommes: incorporer la "bonne » masculinité (hétérosexuelle, en l'occurrence) au bon moment. L'hétérosexualité se révèle ainsi comme une norme plurielle, qui se prête à de nombreuses négociations (Fidolini 2015), donnant vie à différents profils de la masculinité hétéronormative : celui du jeune qui joue le rôle de l'homme sérieux et adulte dans le scénario patriarcal, celui du dragueur de rue, celui de l'ami complice. Toutes ces formes d'expression de la masculinité confirment et réaffirment l'hégémonie de la norme hétérosexuelle, dont la masculinité prédatrice finit par être seulement l'une des manifestations possibles: peut-être la plus spectaculaire, mais certainement pas la seule.

51 De surcroît, la sexualité n'est pas la seule variable qui influence les conduites et les récits de ces jeunes hommes au sein des rapports de pouvoir traversés par chacun. Elle s'imbrique avec différentes relations, intra et intergénérationnelles, et intra et interculturelles.

52 Dans le premier cas d'étude analysé, aussi bien Soufiane que la mère marocaine mobilisent un trait spécifique de la masculinité hétéronormative à travers la référence à l'idéal patriarcal ${ }^{2}$. Tout en appartenant à deux générations différentes, ils s'en servent pour marquer leur appartenance à une culture définie comme " marocaine », qui serait différente de celle des Européen-ne-s, « les autres », et à travers laquelle ils valorisent leur condition minoritaire. De la même manière, cette même masculinité patriarcale, qui s'exprimerait dans le mariage, qui viserait au respect de la femme en tant qu'épouse, et qui est mise en scène par Soufiane vis-à-vis de la mère marocaine et de sa fille, se transforme en masculinité virile et (hétéro)sexuellement débordante dans l'espace de la rue, dans les lieux de la drague, et au sein de l'espace homosocial des pairs.

53 Dans le deuxième cas d'étude, nous observons que le pouvoir de l'hétéronormativité n'est pas utilisé afin de réaffirmer une culture minoritaire vis-à-vis du regard majoritaire, mais devient plutôt un enjeu de la hiérarchisation des rapports entre masculinités minoritaires. Cela est particulièrement évident si l'on pense à la position subordonnée d'Anouar face à son ami Mehmet, jeune homme immigré comme lui, qui semble dominer les relations homosociales et imposer sa masculinité comme hégémonique. De surcroît, cette logique de subordination à la masculinité hégémonique influence aussi les relations entre hommes et femmes: ce n'est pas un 
hasard si Anouar semble craindre l'avis de Mehmet sur la jeune femme de couleur - «la black [...] j'en sais pas ce qu'il en pensera d'une black»-, en interprétant ce trait spécifique de sa partenaire comme un élément susceptible de devenir la cible des jugements de Mehmet.

Comme nous l'avons vu, le pouvoir hiérarchisant de l'hétéronormativité circule à travers les représentations de la sexualité véhiculées par les pairs; il influence les dynamiques de l'identification de genre; il agit au sein des interactions quotidiennes entre hommes et femmes, ou entre hommes et entre femmes; il traverse les relations entre les générations; il contribue à définir les rapports entre sujets ayant les mêmes origines ou ayant des origines différentes. Loin de se limiter à définir le pouvoir hégémonique de l'hétérosexualité et le désir sexuel pour une personne de sexe opposé, l'hétéronormativité est donc une force complexe qui hiérarchise les sexes et les sexualités au sein d'un ensemble plus vaste de relations et de rapports sociaux (Clair, 2008). Les hommes interviewés font de l'hétérosexualité un trait principal de leur masculinité idéale et essaient d'en mettre en scène les traits pour montrer qu'ils sont effectivement des hommes, des « vrais ». Ceux qui n'arrivent pas à accomplir le modèle masculin de référence hétérosexuel en subissent la force hiérarchisante. Dans ce cadre, la masculinité prédatrice se traduit en idéal hégémonique socialement partagé, qui - comme tout autre idéal hégémonique (Connell \& Messerschmidt 2005) - se nourrit des tensions vers sa mise en pratique pour confirmer et renouveler son pouvoir tout en hiérarchisant, en même temps, les rôles et les conduites des acteurs qu'il produit.

L'auteur remercie vivement les relecteurs de l'article et les membres du comité de rédaction de

la revue pour leurs remarques, qui ont grandement contribué à enrichir la version finale de ce texte.

\section{BIBLIOGRAPHIE}

ANDERSON, Eric \& MCCORMACK, Mark. 2015. « Cuddling and Spooning Heteromasculinity and Homosocial Tactility among Student-athletes » Men and Masculinities 18 (2) : 214-230.

BOLOGNE, Jean-Claude. 2010. L'Invention de la drague. Une histoire de la conquête amoureuse. Paris : Éditions du Seuil.

BOUHDIBA, Abdelwahab. 1975. La Sexualité en Islam. Paris : PUF.

BOURDIEU, Pierre. 2000. «La Maison ou le monde renversé », in Esquisse d'une théorie de la pratique. Précédé de Trois études d'ethnologie kabyle, BOURDIEU, Pierre. Paris : Éditions du Seuil, 61-82.

BOZON, Michel. 1995. « Observer l'inobservable : la description et l'analyse de l'activité sexuelle ", in Sexualité et sida. Recherches en sciences sociales, BAJOS, Nathalie et al. (éd.). Paris : Agence nationale de la recherche sur le sida, 39-56. 
BUTLER, Judith. 2009. Ces Corps qui comptent. De la matérialité et des limites discursives du «sexe »

(C. Nordmann, trad.). Paris : Éditions Amsterdam.

COATES, Jennifer. 2003. Men talk. Oxford : John Wiley \& Sons.

CLAIR, Isabelle. 2008. Les Jeunes et l'amour dans les cités. Paris : Armand Colin.

CONNELL, Raewyn. 2005 [1993]. Masculinities. Berkeley : University of California Press.

CONNELL, Raewyn \& MESSERSCHMIDT, James W. 2005. « Hegemonic Masculinity : Rethinking the Concept » Gender \& Society 19 (6) : 829-859.

DEMETRIOU, Demetrakis Z. 2015. «La Masculinité hégémonique : lecture critique d'un concept de Raewyn Connell » Genre, Sexualité \& Société [en ligne] 13, consulté le 17 novembre 2017. URL : http://gss.revues.org/3546

DIALMY, Abdessamad. 2009. Vers une nouvelle masculinité au Maroc. Dakar : CODESRIA.

DURET, Pascal. 1999. Les Jeunes et l'identité masculine. Paris : PUF.

FERRERO CAMOLETTO, Raffaella \& BERTONE, Chiara. 2009. « Like a sex machine ? La naturalizzazione della sessualità maschile ", in Uomini e corpi. Una riflessione sui rivestimenti della mascolinità, RUSPINI, Elisabetta (éd.). Milan : FrancoAngeli, 133-150.

FIDOLINI, Vulca. 2014. «L'Honneur, outil de la construction identitaire. Masculinités, sexualité et altérité » REALIS - Revista de Estudos AntiUtilitaristas e PosColoniais 4 (1) : 117-138.

FIDOLINI, Vulca. 2015. Les Constructions de l'hétéronormativité. Sexualité, masculinités et transition vers l'âge adulte chez de jeunes Marocains en Europe. Thèse de doctorat en sociologie. Strasbourg :

Université de Strasbourg.

FIDOLINI, Vulca. 2017. « Habiter l'ordre hétéronormatif et la masculinité par le mariage » Genre, Sexualité \& Sociétés [en ligne] 17, consulté le 17 novembre 2017. URL : https://gss.revues.org/3993

FLOOD, Michel. 2008. « Men, Sex and Homosociality. How Bonds between Men Shape Their Sexual Relations with Women » Men and Masculinities, 10- $3: 339-359$.

GOFFMAN, Erving. 1987. La Mise en scène de la vie quotidienne. La présentation de soi. Paris : Éditions de Minuit.

GOUYON, Marien. 2010. « Parcours homosexuels du Maroc à la Belgique : l'émigration comme moyen d'émancipation? » L'Autre 11 (3) : 310-320.

GOURARIER, Mélanie. 2017. Alpha Mâle. Séduire les femmes pour s'apprécier entre hommes. Paris : Seuil.

GRAMSCI, Antonio. 2012. Guerre de mouvement et guerre de position, textes choisis et présentés par Razmig Keucheyan. Paris : La Fabrique.

GUÉNIF-SOUILAMAS, Nacira. 2004. «De nouveaux ennemis intimes : le garçon arabe et la fille beurette ", in Les féministes et le garçon arabe, GUÉNIF-SOUILAMAS, Nacira \& MACÉ, Éric. La Tour d'Aigues : Éditions de l'Aube, 59-95.

KIESLING, Scott Fabius. 2005. « Homosocial desire in men's talk : Balancing and re-creating cultural discourses of masculinity » Language in Society 34 (5) : 695-726.

LA CECLA, Franco. 2002. Ce qui fait un homme. Paris : Liana Lévi.

LEBRUN, Aurélie. 2003. Prendre et trouver sa place : discours hétéronormatifs et pratiques hétérosexuelles dans un cruising bar de Montréal. Thèse de $\mathrm{PhD}$ en géographie. Montréal : McGill University. 
MAUSS, Marcel. 1950. «Les Techniques du corps ", in Sociologie et anthropologie, MAUSS, Marcel.

Paris : PUF, 364-386.

OUZGANE Lahoucine. 2003. «Islamic Masculinities : an Introduction », Men and Masculinities 5 (3) : 231-235.

REBUCINI, Gianfranco. 2009. Les Masculinités au Maroc. Pour une anthropologie des genres et des sexualités dans la ville de Marrakech. Thèse de doctorat en anthropologie sociale et ethnologie. Paris : École des hautes études en sciences sociales.

SAADI-MOKRANE, Djamila. 2004. « Petit lexique du dragueur algérois », in La Virilité en Islam, BENSLAMA, Fethi \& TAZI, Nadia (éd.). La Tour d'Aigues : Éditions de l'Aube, 181-186.

WHITEHEAD, Stephen M. 2002. Men and Masculinities: Key Themes and New Directions. Cambridge : Polity Press.

\section{NOTES}

1. Ville frontière avec l'Allemagne, qui se trouve à quelques kilomètres de Strasbourg.

2. L'expression « idéal » ne définit pas une forme parfaite, idéalisée et suprême de la masculinité, mais elle indique plutôt le pouvoir persuasif d'un modèle masculin dont les traits constitutifs orientent les formes de l'identification de genre chez les jeunes hommes interrogés.

\section{RÉSUMÉS}

En s'appuyant sur les résultats d'une étude qualitative menée auprès de 23 jeunes hommes marocains, immigrés en France, âgés de 20 à 30 ans et musulmans, cet article analyse les modalités de mise en action de la norme hétérosexuelle et son impact sur les processus d'identification de genre. Il accorde une place centrale à l'analyse des récits masculins dans différents contextes, homosociaux - entre hommes - et mixtes, afin de décrire comment la construction de masculinités hégémoniques hétérosexuelles produit d'autres masculinités qui leur sont «complémentaires». L'attention est notamment concentrée sur la production de la «masculinité prédatrice » - à la (hétéro)sexualité active et débordante - et des masculinités "complices». Cette démarche permet d'analyser non seulement les trajectoires de l'identification de genre chez les personnes interviewées, mais aussi de saisir l'impact d'un ensemble de rapports de pouvoir (notamment inter et intra-générationnels et entre populations minoritaire et majoritaire) sur les vécus des jeunes hommes rencontrés.

By exploiting results from a qualitative study carried out in France on 23 young Moroccan and Muslim migrant men, between the ages of 20 and 30, this article analyses how heterosexuality expresses its normative power on gender identification processes. The paper focuses attention on constructions of masculinity in both homosocial and mixed contexts in order to describe how the production of heterosexual masculinities is also based on shaping "complementary" profiles of these hegemonic masculinities. In particular, we deal with the case of "predatory" masculinity as well as "complicit masculinities" which endorse and reaffirm the hegemonic power of the predatory one. This approach leads to analyse not only gender identification trajectories but also 
to observe how intra- and inter- cultural relations (e.g. between migrant and majority populations) or intra- and inter- generational relationships (e.g. between young people and adults) shape the experiences of the interviewees.

INDEX

Thèmes : Recherches

Keywords : heterosexuality, heteronormativity, ethnography, body, critique of social relations Mots-clés : hétérosexualité, hétéronormativité, ethnographie, corps, rapports de pouvoir

\section{AUTEUR}

\section{VULCA FIDOLINI}

Docteur en sociologie, université de Strasbourg, UMR 7367 Dynamiques Européennes Chercheur postdoctoral au laboratoire Dynamiques Européennes (UMR 7367, CNRS - université de Strasbourg), ses travaux portent sur les masculinités et les formes d'expression de l'hétéronormativité en société. Ses recherches les plus récentes articulent les questions de genre, santé et alimentation. 